\section{Istituto}

Nazionale

Fisica

Nucleare
Sezione SANITÀ

Istituto Superiore di Sanità

Viale Regina Elena 299

I-00161 Roma, Italy

INFN-ISS 96/12

December 1996

\title{
LIGHT-FRONT CQM CALCULATIONS OF BARYON ELECTROMAGNETIC FORM FACTORS由
}

\author{
F. Cardarelli ${ }^{(1)}$, E. Pace ${ }^{(2)}$, G. Salmè ${ }^{(3)}$ and S. Simula ${ }^{(3)}$ \\ ${ }^{(1)}$ Dept. of Physics and Supercomputer Computations Research Institute, \\ Florida State University, Tallahassee, FL 32306,USA \\ ${ }^{(2)}$ Dipartimento di Fisica, Università di Roma "Tor Vergata" and \\ Istituto Nazionale di Fisica Nucleare, Sezione Tor Vergata, \\ Via della Ricerca Scientifica 1, I-00133 Roma, Italy \\ (3) Istituto Nazionale di Fisica Nucleare, Sezione Sanità, \\ Viale Regina Elena 299, I-00161 Roma, Italy
}

\begin{abstract}
The parameter-free predictions for the $N-P_{11}(1440)$ and $N-P_{33}(1232)$ electromagnetic transition form factors, obtained within our light-front constituent quark model using eigenfunctions of a baryon mass operator which includes a large amount of configuration mixing, are reported. The effects due to small components in the baryon wave functions, such as S'- and D-wave, are also investigated.
\end{abstract}

\footnotetext{
${ }^{a}$ To appear in Nuclear Physics A (1997): Proceedings of the International Workshop on Hadron dynamics with the new DA $\phi N E$ and CEBAF facilities, Frascati, Italy, 11-14 November 1996.
} 


\section{INTRODUCTION}

The electromagnetic (e.m.) excitation of Nucleon resonances, in the space-like region, represents a very interesting tool for gathering information on the internal structure of baryons and it is one of the major issues of the TJNAF research programme [1]. For investigating this topic we have developed a phenomenological approach [2], based on a constituent quark $(C Q)$ model which features: i) a proper treatment of relativistic effects, achieved by formulating the model on the light-front $(L F)$, see e.g. [3]; ii) baryon eigenfunctions of a mass operator that describes fairly well the mass spectrum [ [i], at variance with the widely adopted gaussian-like ansatz, see e.g. Ref. [5]; iii) a one-body approximation for the e.m. current able to reproduce the experimental data on the Nucleon and pion form factors.

\section{GENERAL FORMALISM}

In the $L F$ hamiltonian dynamics [3] a baryon state, in the $u-d$ sector, $\left|\Psi_{J J_{n} \pi}^{T T_{3}}, \tilde{P}\right\rangle$, is an eigenstate of: i) isospin, $T$ and $T_{3}$; ii) parity, $\pi$; iii) kinematical (non-interacting) $L F$ angular momentum operators $j^{2}$ and $j_{n}$, where the vector $\hat{n}=(0,0,1)$ defines the spin quantization axis; iv) total $L F$ baryon momentum $\tilde{P} \equiv\left(P^{+}, \vec{P}_{\perp}\right)=\tilde{p}_{1}+\tilde{p}_{2}+\tilde{p}_{3}$, where $P^{+}=P^{0}+\hat{n} \cdot \vec{P}$ and $\vec{P}_{\perp} \cdot \hat{n}=0$. The state $\left|\Psi_{J J_{n} \pi}^{T T_{3}}, \tilde{P}\right\rangle$ factorizes into $\left|\Psi_{J J_{n} \pi}^{T T_{3}}\right\rangle|\tilde{P}\rangle$, and the intrinsic part $\left|\Psi_{J J_{n} \pi}^{T T_{3}}\right\rangle$ can be constructed from the eigenstate $\left|\psi_{J J_{n} \pi}^{T T_{3}}\right\rangle$ of the canonical angular momentum, i.e. $\left|\Psi_{J J_{n} \pi}^{T T_{3}}\right\rangle=\mathcal{R}^{\dagger}\left|\psi_{J J_{n} \pi}^{T T_{3}}\right\rangle$, where the unitary operator $\mathcal{R}^{\dagger}=\prod_{j=1}^{3} R_{M e l}^{\dagger}\left(\vec{k}_{j}, m_{j}\right)$, with $R_{\text {Mel }}\left(\vec{k}_{j}, m_{j}\right)$ being the generalized Melosh rotation [3]. Then $\left|\psi_{J_{n} \pi}^{T T_{3}}\right\rangle$ satisfies the following mass equation

$$
\left(M_{0}+V\right)\left|\psi_{J J_{n} \pi}^{T T_{3}}\right\rangle=M\left|\psi_{J J_{n} \pi}^{T T_{3}}\right\rangle
$$

where $M_{0}=\sum_{i=1}^{3} \sqrt{m_{i}^{2}+\vec{k}_{i}^{2}}$ is the free mass operator, $m_{i}$ the $C Q$ mass $\left(m_{u}=m_{d}=\right.$ $0.220 \mathrm{GeV}$ accordingly to [4] $), M$ the baryon mass, and $J(J+1), J_{n}$ are the eigenvalues of the operators $j^{2}, j_{n}$, respectively. The interaction $V$ has to be independent of the total momentum $P$ and invariant upon spatial rotations and translations. We can identify Eq. (11) with the baryon mass equation proposed by Capstick and Isgur $(C I)$ 田. Their $C Q$ interaction is composed by a linear confining term, dominant at large separations, and a onegluon-exchange $(O G E)$ term, dominant at short separations, given by a central Coulomb-like potential and a spin-dependent part, responsible for the hyperfine splitting of baryon masses. The mass equation (11) has been accurately solved by expanding the eigenstates onto a large harmonic oscillator $(H O)$ basis (up to $20 \mathrm{HO}$ quanta) and then by applying the RayleighRitz variational principle.

The $C Q$ momentum distribution calculated from the baryon eigenfunctions of Eq. (11), with the $C I$ interaction, has a striking feature [2]: for a $C Q$ three-momentum larger than $1 \mathrm{GeV} / \mathrm{c}$, it is order of magnitude larger than momentum distributions evaluated from model functions, such as gaussian or power-law fiunctions (cf. [5]). This fact is due to the smoothly singular $O G E$ part and has immediate consequences on the interpretation of the 
resonances, for instance, the Roper resonance is not a simple (first) radial excitation of the Nucleon and it has a large mixed-symmetry S'component $\left(P_{S^{\prime}}^{\text {Roper }}=9.3 \%\right)$.

In the $L F$ formalism the space-like e.m. form factors are related to the matrix elements of the plus component of the current, $\mathcal{I}^{+}=\mathcal{I}^{0}+\hat{n} \cdot \overrightarrow{\mathcal{I}}$, with the standard choice $q^{+}=q^{0}+\hat{n} \cdot \vec{q}=$ $P_{f}^{+}-P_{i}^{+}=0$, that allows to suppress the contribution of the pair creation from the vacuum [6]. For a $\frac{1}{2}^{+}$baryon in the final state, e.g. the Nucleon $(f=N)$ or the Roper resonance $(f=R)$, the Dirac and Pauli form factors, $F_{1(2)}^{f \tau}\left(Q^{2}\right)(\tau=p$ or $n)$, are given by

$$
F_{1}^{f \tau}\left(Q^{2}\right)=\frac{1}{2} \operatorname{Tr}\left[\mathcal{I}^{+}(\tau)\right] \quad, \quad F_{2}^{f \tau}\left(Q^{2}\right)=i \frac{M_{f}+M_{N}}{2 Q} \operatorname{Tr}\left[\sigma_{2} \mathcal{I}^{+}(\tau)\right]
$$

with $\mathcal{I}_{\nu_{f} \nu}^{+}(\tau)=\bar{u}_{L F}\left(\tilde{P}_{f}, \nu_{f}\right)\left\{F_{1}^{f \tau}\left(Q^{2}\right) \gamma^{+}+F_{2}^{f \tau}\left(Q^{2}\right) i \sigma^{+\rho} q_{\rho} /\left(M_{f}+M_{N}\right)\right\} u_{L F}\left(\tilde{P}_{i}, \nu\right)$, where $Q^{2} \equiv-q \cdot q$ is the squared four-momentum transfer, $\sigma^{+\rho}=\frac{i}{2}\left[\gamma^{+}, \gamma^{\rho}\right], u_{L F}\left(\tilde{P}_{i}, \nu\right)\left[u_{L F}\left(\tilde{P}_{f}, \nu_{f}\right)\right]$ the Nucleon [final baryon] LF-spinor, $\sigma_{2}$ a Pauli matrix. For the excitation to a $\Delta$ resonance, or in general to a $\frac{3}{2}^{+}$baryon, the kinematic-singularity free form factors $G_{1,2,3}$ [7] are related to the LF matrix elements of $\mathcal{I}^{+}$as follows

$$
\begin{aligned}
\mathcal{I}_{\frac{3}{2} \frac{1}{2}}^{+} & =\frac{Q}{\sqrt{2}}\left[G_{1}\left(Q^{2}\right)+\frac{M_{\Delta}-M_{N}}{2} G_{2}\left(Q^{2}\right)\right] \\
\mathcal{I}_{\frac{1}{2} \frac{1}{2}}^{+} & =-\frac{Q^{2}}{\sqrt{6}}\left[\frac{G_{1}\left(Q^{2}\right)}{M_{\Delta}}+\frac{G_{2}\left(Q^{2}\right)}{2}-\frac{M_{\Delta}-M_{N}}{M_{\Delta}} G_{3}\left(Q^{2}\right)\right] \\
\mathcal{I}_{\frac{1}{2}-\frac{1}{2}}^{+} & =\frac{Q}{\sqrt{6}}\left[G_{1}\left(Q^{2}\right) \frac{M_{N}}{M_{\Delta}}-\frac{M_{\Delta}-M_{N}}{2 M_{\Delta}} G_{2}\left(Q^{2}\right)-\frac{Q^{2}}{M_{\Delta}} G_{3}\left(Q^{2}\right)\right] \\
\mathcal{I}_{\frac{3}{2}-\frac{1}{2}}^{+} & =-\frac{Q^{2}}{2 \sqrt{2}} G_{2}\left(Q^{2}\right)
\end{aligned}
$$

with $\mathcal{I}^{+}{ }_{\nu_{f} \nu}(\tau)=\left\langle\frac{1}{2} \tau, 10 \mid T T_{3}\right\rangle \bar{w}_{L F}^{\mu}\left(\tilde{P}_{f}, \nu_{f}\right) \Gamma_{\mu}^{+} u_{L F}\left(\tilde{P}_{i}, \nu\right)$, with $\Gamma_{\mu}^{+}=G_{1}\left(Q^{2}\right) \mathcal{K}_{1 \mu}^{+}+G_{2}\left(Q^{2}\right)$ $\mathcal{K}_{2 \mu}^{+}+G_{3}\left(Q^{2}\right) \mathcal{K}_{3 \mu}^{+}$(tensors $\mathcal{K}_{i \mu}{ }^{\rho}$ are defined in [7]). Differently from the case of a $\frac{1}{2}^{+}$baryon, the number of form factors for the excitation of a $\frac{3}{2}^{+}$baryon is not equal to the one of the LF matrix elements of $\mathcal{I}^{+}$, cf. Eq. (3). For the exact current the inversion of Eq. (3) is unique. Since we adopt a one-body approximation for $\mathcal{I}^{+}$(see below) different choices of LF matrix elements can lead to different predictions for the $G_{i}$ form factors. In the actual calculation for the N- $\Delta$ transition we consider two different prescriptions for extracting the $G_{i}$ form factors from Eq. (3): i) $G_{1}$ and $G_{3}$ are obtained from the first three equations in (3), while $G_{2}$ is directly taken from the fourth one (prescription I); ii) all the $G_{i}$ form factors are extracted from the first three equations (prescription II).

\section{RESULTS}

Elastic and transition form factors have been evaluated using eigenvectors of Eq. (1) and approximating the $\mathcal{I}^{+}$component of the e.m. current by the sum of one-body $C Q$ currents 
(see [国), i.e.

$$
\mathcal{I}^{+}(0) \approx \sum_{j=1}^{3} I_{j}^{+}(0)=\sum_{j=1}^{3}\left(e_{j} \gamma^{+} f_{1}^{j}\left(Q^{2}\right)+i \kappa_{j} \frac{\sigma^{+\rho} q_{\rho}}{2 m_{j}} f_{2}^{j}\left(Q^{2}\right)\right)
$$

where $e_{j}\left(\kappa_{j}\right)$ is the charge (anomalous magnetic moment) of the j-th quark, and $f_{1(2)}^{j}$ the corresponding Dirac (Pauli) form factor. Though the hadron e.m. current has to contain many-body components for fulfilling both gauge and rotational invariances, we have shown in [2] (a) that the $C Q$ form factors can be chosen so that the effective one-body current (4) is able to give a coherent and accurate description of both the pion and Nucleon experimental form factors. After fixing the $C Q$ form factors, we have calculated, without free parameters, the N-Roper helicity amplitudes $A_{\frac{1}{2}}^{p(n)}\left(Q^{2}\right)$ and $-S_{\frac{1}{2}}^{p(n)}\left(Q^{2}\right)$, shown in Fig. 1 (see [2] (c)). Our results both for $A_{\frac{1}{2}}^{p(n)}\left(Q^{2}\right)$ and $S_{\frac{1}{2}}^{p}\left(Q^{2}\right)$ exhibit a remarkable reduction (bringing our predictions closer to the experimental analyses [9, 10]) with respect to non-relativistic [8] as well as relativistic [5 predictions, based on simple gaussian-like wave functions. It is worth noting that the helicity amplitudes $S_{\frac{1}{2}}^{p}\left(Q^{2}\right)$ and $S_{\frac{1}{2}}^{n}\left(Q^{2}\right)$ are sizably sensitive to the presence of the mixed-symmetry $S^{\prime}$ component in the $C I$ wave function.

The magnetic form factor $G_{M}^{N-\Delta}\left(Q^{2}\right)$ and the ratios $E_{1} / M_{1}=-G_{E}^{N-\Delta}\left(Q^{2}\right) / G_{M}^{N-\Delta}\left(Q^{2}\right)$ and $S_{1} / M_{1}=-\left(\sqrt{K^{+} K^{-}} / 4 M_{\Delta}^{2}\right) G_{C}^{N-\Delta}\left(Q^{2}\right) / G_{M}^{N-\Delta}\left(Q^{2}\right)$ (see Ref. [7] for the relation with the $G_{i}$ form factors), calculated within our model without free-parameters (see also [2](b)) are shown in Fig. 2 (a,b,c), respectively. The differences between the prescription I and II are not so relevant for $G_{M}^{N-\Delta}$ as they are for $E_{1} / M_{1}$ and $S_{1} / M_{1}$; however the effect due to the $\mathrm{D}$-wave component is always small for both prescriptions (indeed $P_{D}^{\Delta}=1.1 \%$ ). Although two-body $C Q$ currents are lacking in the approximation (田) it is encouraging that our effective current can provide an overall description of relatively small quantities, such as the ratios $E_{1} / M_{1}(\approx 5 \%)$ and $S_{1} / M_{1}(\approx 10 \%)$ for $Q^{2}$ up to few $G e V / c^{2}$. Finally in Fig. 2 (d) the ratio of $G_{M}^{N-\Delta}\left(Q^{2}\right)$ (obtained in prescription II) and the isovector part of the Nucleon magnetic form factor, $G_{M}^{p}\left(Q^{2}\right)-G_{M}^{n}\left(Q^{2}\right)$, is shown. It can clearly be seen that this ratio is largely insensitive to the presence of $C Q$ form factors, whereas it is sharply affected by the spin-dependent part of the $C I$ potential, which is generated by the chromomagnetic interaction.

In conclusion, we have reported the calculations of the e.m. form factors for the transition $N-P_{11}(1440)$ and $N-P_{33}(1232)$, obtained within our approach based on a relativistic $C Q$ model, baryon eigenfunctions of a mass operator and an effective one-body $C Q$ current. The results allow an overall description of the data, but they also indicate the necessity of the introduction of at least a two-body term in the $C Q$ current, in order to give accurate predictions for "small" form factors (like, e.g. $E_{1}$ or $S_{1}$ for the N- $\Delta$ transition). Finally, we have shown that the determination of $G_{M}^{N-\Delta}\left(Q^{2}\right) /\left(G_{M}^{p}\left(Q^{2}\right)-G_{M}^{n}\left(Q^{2}\right)\right)$ could provide relevant information on $\mathrm{SU}(6)$ breaking effects in $\mathrm{N}$ and $\Delta$ wave functions, without substantial model dependence. 


\section{ACKNOWLEDGEMENT}

One of the authors (F.C.) acknowledges a warm hospitality by Simon Capstick and the partial support by the U.S. Department of Energy through Contract DE-FG05-86ER40273, and by the Florida State University Supercomputer Computations Research Institute (SCRI) which is partially funded by the Department of Energy through Contract DE-FC05-85ER250000.

\section{References}

[1] For a review of the $N^{*}$ program at $T J N A F$, see, e.g., V.D. Burkert, Proc. of the XIV Int. Conf. on Few-Body Problems in Physics, Williamsburg (USA), May 1994, ed. F. Gross, AIP Conf. Proc. 334 (1995) 127.

[2] a) F. Cardarelli, E. Pace, G. Salmè and S.Simula, Phys. Lett. B357 (1995) 267; FewBody Systems Suppl. 8 (1995) 345; b) F. Cardarelli, E. Pace, G. Salmè and S. Simula, Phys. Lett. B371 (1996) 7, and to be published; c) F. Cardarelli, E. Pace, G. Salmè and S. Simula, nucl-th 9609047, submitted to Phys. Lett. B.

[3] For a review, see B.D. Keister and W.N. Polyzou, Adv. in Nucl. Phys. 20 (1991) 225 and F. Coester, Progress in Part. and Nucl. Phys. 29 (1992) 1.

[4] S. Capstick and N. Isgur, Phys. Rev. D34 (1986) 2809.

[5] S. Capstick and B. Keister, Phys. Rev. D51 (1995) 3598.

[6] See, e.g., L.L. Frankfurt and M.I. Strikman, Nucl. Phys. B 148 (1979) 107.

[7] R.C.E. Devenish, T.S. Eisenschitz and J.G. Korner, Phys. Rev. D14 (1976) 3063.

[8] Z. Li, V. Burkert and Z. Li, Phys. Rev. D46 (1992) 70.

[9] Particle Data Group, R.M. Barnett et al., Phys. Rev. D54 (1996) 1.

[10] (a) C. Gerhardt, Z. Phys. C4 (1980) 311; (b) B. Boden and G. Krosen, in Proceedings of the Conference on Research Program at CEBAF II, Report of the 1986 Summer Study Group, edited by V. Burkert et al., CEBAF (USA), 1986.

[11] (a) W. Bartel et al., Phys. Lett. B 28 (1968) 148; (b) P. Stoler, Phys. Rep. 226 (1993) 103; (c) C. Keppel, in "CEBAF at Higher Energies", eds. N. Isgur and P. Stoler (CEBAF, Newport News, April 1994) p. 237; d) R.M. Davidson and N.C. Mukhopadhyay, Phys. Lett. B 353 (1995) 131.

[12] (a) J.C. Alder et al., Nucl. Phys. B46 (1972) 573; (b) R. Siddle et al., Nucl. Phys. B35 (1971) 93; (c) V.D. Burkert and L. Elouadrhiri, Phys. Rev. Lett. 75 (1995) 3614, and private communication. 

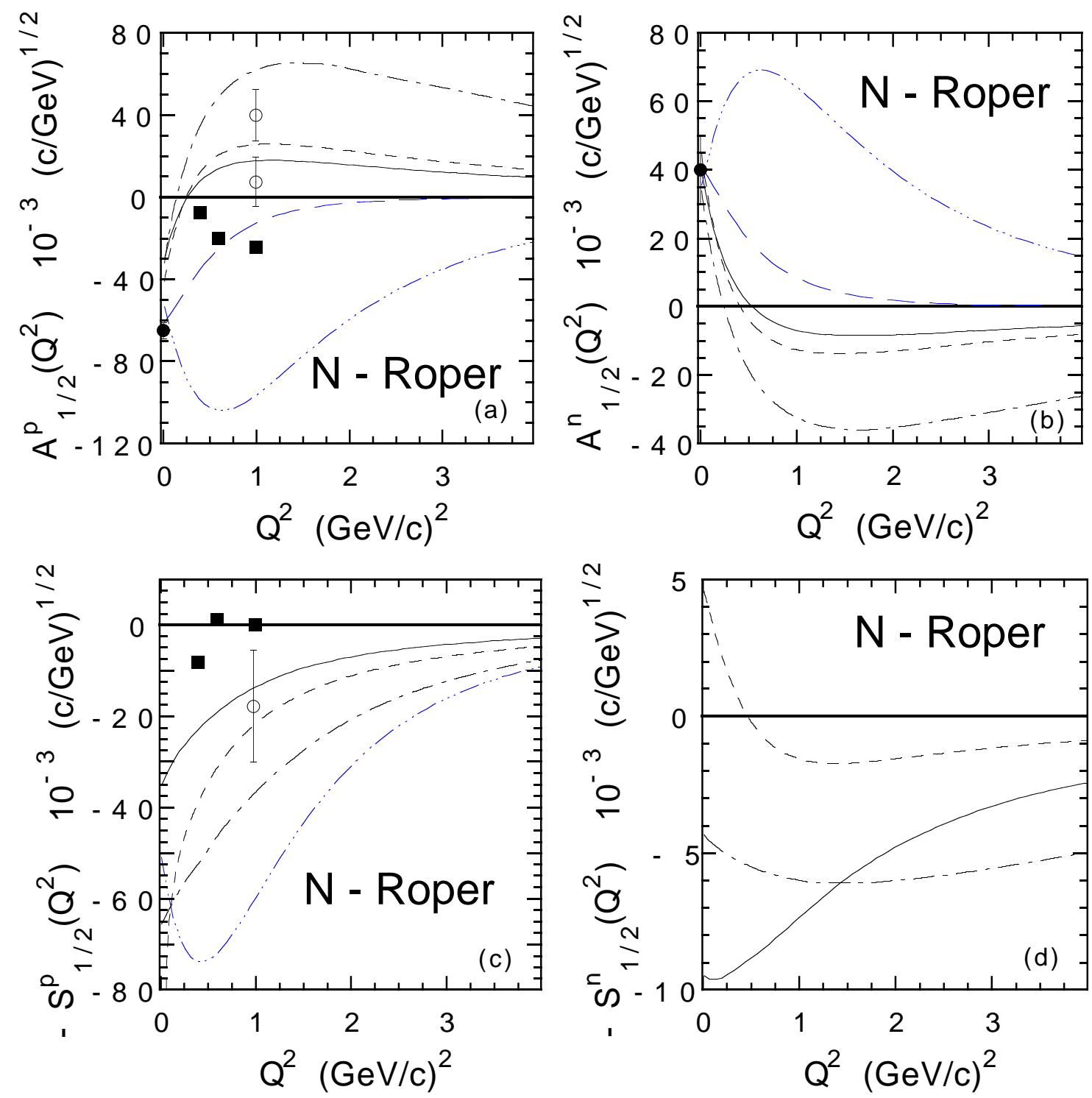

Figure 1. The $N-P_{11}(1440)$ helicity amplitudes $A_{\frac{1}{2}}^{p(n)}\left(Q^{2}\right)$ and $-S_{\frac{1}{2}}^{p(n)}\left(Q^{2}\right)$ vs. $Q^{2}$. Solid line: LF calculation obtained by using baryon wave functions corresponding to the $C I$ interaction [4] and the e.m. current (画) with the $C Q$ form factors determined in [a](a); dashed line: the same as the solid one, but without the S' component in the baryon eigenfunctions; dotdashed line: LF calculation obtained by using the gaussian functions of Ref. [5] without CQ form factors. The long-dashed and triple-dot-dashed lines correspond to the non-relativistic $q^{3} G$ and $q^{3}$ models of Ref. [8]. Full dots: $P D G$ values [9]; full squares and open dots: phenomenological analyses of Ref. [10] (a) and (b), respectively. Within the hybrid $q^{3} G$ model $S_{\frac{1}{2}}^{p(n)}\left(Q^{2}\right)=0$, whereas within the non-relativistic $q^{3}$ model only $S_{\frac{1}{2}}^{n}\left(Q^{2}\right)=0$. (After [2] (c)) 

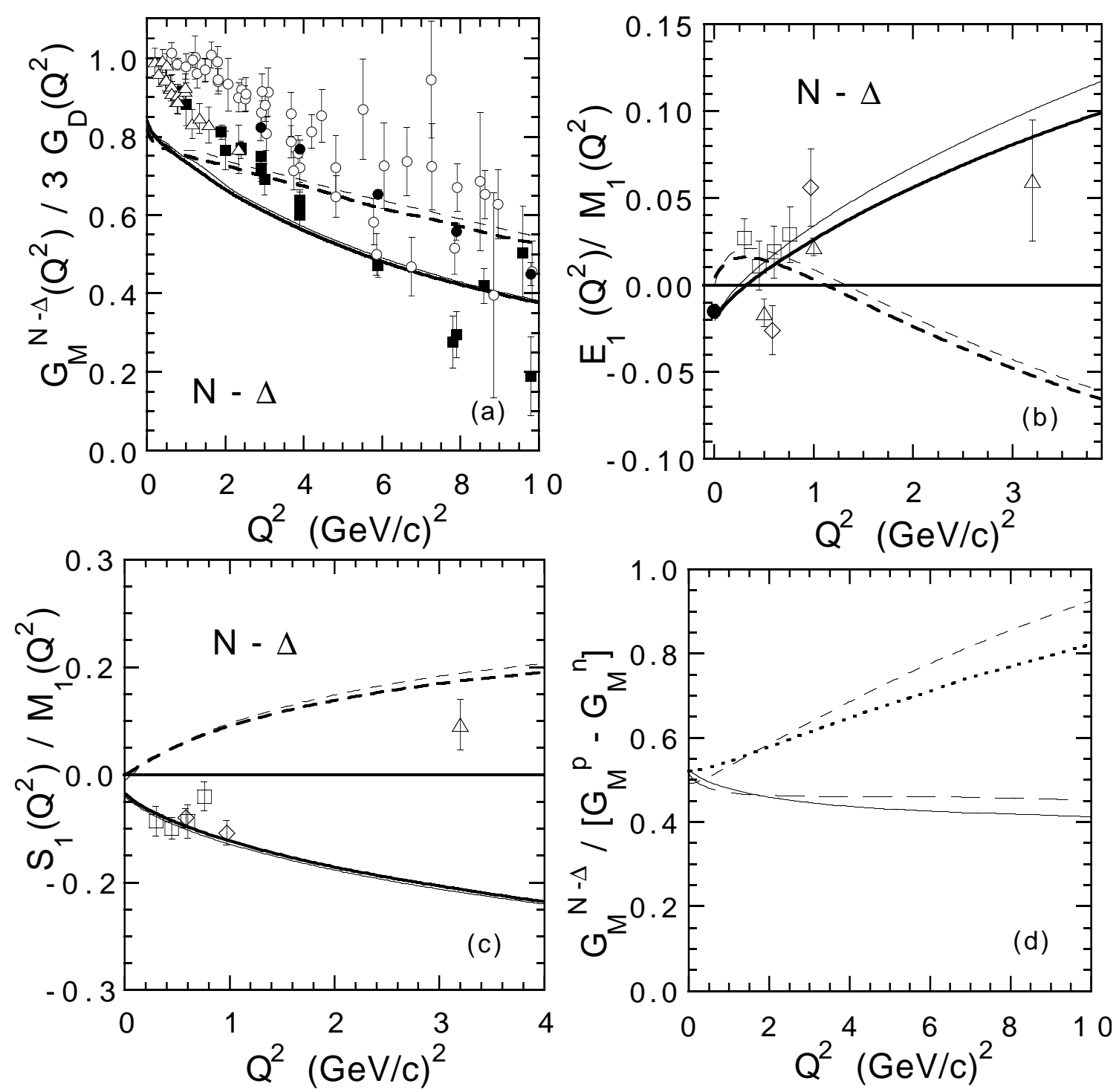

Figure 2. (a) $G_{M}^{N-\Delta}\left(Q^{2}\right) / 3 G_{D}\left(Q^{2}\right)$, with $G_{D}\left(Q^{2}\right)=1 /\left(1+Q^{2} / 0.70\right)^{2}$, vs $Q^{2}$. The thick and thin lines correspond to the LF calculations with and without the D-wave in the $\Delta$ eigenstate. The e.m. current (4) with the $C Q$ form factors determined in [2] (a) has been adopted. Solid lines: prescription I (see text). Dashed lines: prescription II (see text). Triangles: [11] (a); full squares: [11](b); open dots: [11](c); full dots: [11](d). - (b) The same as in (a), but for $E_{1} / M_{1}$. Full dots: PDG [9]; diamonds: [12](a); open squares: [12](b); triangles: [12] (c). - (c) The same as in (b) but for $S_{1} / M_{1}$. - (d) The ratio $G_{M}^{N-\Delta}\left(Q^{2}\right) /\left(G_{M}^{p}\left(Q^{2}\right)-G_{M}^{n}\left(Q^{2}\right)\right)$ vs $Q^{2}$. Solid line: our calculation (prescription II) with the $C I$ baryon eigenfunctions and $C Q$ form factors; dashed line: the same as the solid line, but without $C Q$ form factors; short-dashed line: the same as the dashed line, but with the baryon eigenfunctions corresponding to the spin-independent part of the $C I$ interaction [1]; dotted line: the same as the dashed line, but retaining only the confining part of the $C I$ potential. (After [2] (b)). 